\title{
Sleep and glycemic control in type 1 diabetes
}

Mark Thomaz Ugliara Barone 1,2, Daniela Wey ${ }^{3}$, Fabiola Schorr 4 , Denise Reis Franco ${ }^{2.5}$, Mario Kehdi Carra', Geraldo Lorenzi-Filho ${ }^{4}$, Luiz Menna-Barreto7

\begin{abstract}
Objective: Our aim in the present study was to elucidate how type 1 diabetes mellitus (T1DM) and sleep parameters interact, which was rarely evaluated up to the moment. Materials and methods: Eighteen T1DM subjects without chronic complications, and 9 control subjects, matched for age and BMI, were studied. The following instruments used to evaluate sleep: the Epworth Sleepiness Scale, sleep diaries, actimeters, and polysomnography in a Sleep Lab. Glycemic control inT1DM individuals was evaluated through: $\mathrm{A} 1 \mathrm{C}$, home fingertip glucometer for 10 days (concomitant with the sleep diary and actimeter), and CGM or concomitant with continuous glucose monitoring (during the polysomnography night). Results: Comparing with the control group, individuals with diabetes presented more pronounced sleep extension from weekdays to weekends than control subjects $(p=0.0303$ ). Among T1DM, glycemic variability (SD) was positively correlated with sleep latency $(r=0.6525$, $p=0.0033)$; full awakening index and arousal index were positively correlated with $A 1 C(r=0.6544$ $p=0.0081$; and $r=0.5680, p=0.0272$, respectively); and mean glycemia values were negatively correlated with sleep quality in T1DM individuals with better glycemic control (mean glycemia $<154 \mathrm{mg} / \mathrm{dL}$ ). Conclusion: Our results support the hypothesis of an interaction between sleep parameters and T1DM, where the glycemic control plays an important role. More studies are needed to unveil the mechanisms behind this interaction, which may allow, in the future, clinicians and educators to consider sleep in the effort of regulating glycemic control. Arch Endocrinol Metab. 2015;59(1):71-8
\end{abstract}

Keywords

Type 1 diabetes mellitus; sleep; blood glucose; sleep-wake cycle disorders

\author{
1 Instituto de Ciências Biomédicas, \\ Universidade de São Paulo (ICB/ \\ USP), São Paulo, SP, Brazil \\ ${ }^{2}$ Associação de Diabetes \\ Juvenil (ADJ Diabetes Brasil), \\ São Paulo, SP, Brazil \\ ${ }^{3}$ Faculdade de Saúde Pública, \\ Universidade de São Paulo (FSP/ \\ USP), São Paulo, SP, Brazil \\ ${ }^{4}$ Instituto do Coração, Faculdade \\ de Medicina, Universidade \\ de São Paulo (InCor/FMUSP), \\ São Paulo, SP, Brazil \\ ${ }^{5}$ Centro de Pesquisas Clínicas \\ (CPClin), São Paulo, SP, Brazil \\ ${ }^{6}$ Hospital das Clínicas, FMUSP, \\ São Paulo, SP, Brazil \\ ${ }^{7}$ Escola de Artes, Ciências \\ e Humanidades (EACH), \\ USP, São Paulo, SP, Brazil \\ Correspondence to: \\ Mark Thomaz Ugliara Barone \\ Instituto de Ciências Biomédicas, \\ Universidade de São Paulo, \\ Departamento de Fisiologia e \\ Biofísica \\ Av. Prof. Lineu Prestes, 1524 (ICB I) \\ 05508-900 - São Paulo, SP, Brazil \\ markbarone@bol.com.br \\ Received on Jun/11/2014 \\ Accepted on Oct/24/2014 \\ DOI: 10.1590/2359-3997000000013
}

\section{INTRODUCTION}

$\mathrm{T}$ here is growing evidence that sleep disorders and sleep loss affect glucose metabolism and insulin resistance (1-5). At the same time, altered glucose metabolism may impact sleep quality $(6,7)$. Relationships between type 2 diabetes mellitus (T2DM) and sleep disordered breathing have been extensively studied. Patients with T2DM present an extremely high prevalence of obstructive sleep apnea, which in turn contributes to poor glycemic control $(6,8)$. In contrast, the relationship between type 1 diabetes mellitus (TIDM) and sleep has not been frequently investigated.

In the present study we evaluate a possible interaction between TIDM and sleep, as proposed before (9). We performed this study in order to broaden the understanding of this vicious circle. Differences between sleep variables in people with TIDM and their peers have already been shown (10). Furthermore, the glycemic control was shown to affect sleep in T1DM, and apnea was positively associated with glycated hemoglobin (AlC) and with the disease duration $(11,12)$. Moreover, nocturnal hypoglycemia was associated with sleep deepening, whereas glycemic variation has been considered to cause awakenings among TIDM children (12). In addition, authors comparing TIDM adults with controls reported that TIDM subjects presented changes in sleep architecture, with increased proportion of stage 2 , besides higher levels of epinephrine and growth hormone during the entire night, and higher ACTH and cortisol during part of the first half of the night (13). As a consequence, TIDM subjects report less restorative sleep (13), and present higher risk for obstructive sleep apnea and poorer sleep quality than control subjects (14). 
Therefore, considering the evidence that sleep quality and duration may affect glucose metabolism $(6,15)$, and with the evidence presented above that sleep quality and duration may be altered in TIDM, we evaluated several sleep and sleep related parameters to broaden the understanding associations of glycemic control and sleep parameters in TIDM patients. Our hypothesis is that poor glycemic control may impact several sleep parameters, including: architecture, quality, and duration. At the same time, sleep impairments may hinder glycemic control in TIDM patients.

\section{MATERIALS AND METHODS}

\section{Subjects}

Eighteen young adults with TIDM, free of diabetes chronic complications, and not taking drugs that could affect sleep, were recruited. They were no night or shift workers and had no previous diagnosis of sleep disorders. In order to select individuals without chronic complications, all TIDM volunteers were subjected to retinal inspection by ophthalmologists, measurement of $24 \mathrm{~h}$ microalbuminuria and creatinin levels, $10 \mathrm{~g}$ monofilament sensation, vibration perception with a $128-\mathrm{Hz}$ tuning fork, resting heart rate, and pressure adaptation when standing up. Individuals presenting impairment in any of these tests, interpreted according to ADA's Standards of Medical Care in Clinical in Diabetes (16), were not included in the study.

Nine control subjects, matched for age and BMI, free of drugs with effect on sleep, no night or shift workers, and without previous diagnosis of sleep disorders, were recruited. Even though these individuals were not obese and had no diabetes symptoms, all of them did a blood glucose test after fasting of 8 hours at a reference laboratory.

\section{Instruments}

Data from all individuals were collected with sleep diaries and actimeters (Tempatilumi, wrist accelerometer produced by CEBrasil) simultaneously during 10 consecutive days, between the end of February and beginning of March. The actimeter was continuously worn on the non-dominant wrist, removed only for shower or any water activity. All volunteers were instructed to press the actimeter event button immediately before removing it, in order to identify unworn periods. During these 10 days, TIDM individuals performed 6-10 home blood glucose tests a day, with a Performa ${ }^{\circledR}$ glucometer (Roche Diagnostics, Mannheim, Germany). The sleep diary was adapted from the Pittsburgh Sleep Diary Wake Time (17), excluding mood and alertness items. Sleep quality was reported by individuals upon awakening also using a Visual Analogue Scale - VAS (18). For the individuals with diabetes a question about hypoglycemia and hyperglycemia during the night and the glycemic value, if measured, was included in the diary. In addition, all volunteers were subjected to: 1 night of polysomnography, and the Epworth Sleepiness Scale (ESS). TIDM subjects wore a Continuous Glucose Monitoring System $\left(\right.$ CGMS $^{\circledast}$, Medtronic) during the polysomnography (PSG); and had their AlC measured after the collection of all other data. The polysomnography equipment used was the EMBLA (Flagra hf. Medical Devices, Reykjavik, Iceland), performed in a blinded manner, at the InCor-HCFMUSP Sleep Lab. Monitoring included 4 channels of electroencephalogram, 2 channels of electrooculogram, besides channels for: electrocardiogram, chin and anterior tibial electromyogram, pulse oxymetry, airflow (oronasal thermistor and cannula pressure), and chest and abdominal straps.

\section{Analysis}

In order to perform some of the analyses, the TIDM group was divided two groups according to the individual mean glycemia $(\mathrm{MG})$, considering all the measurements performed during the 10 consecutive days. The 9 individuals with mean glycemia below $154 \mathrm{mg} / \mathrm{dL}$ (which corresponds to an AlC of $7.0 \%(53 \mathrm{mmol} /$ mol) (19) composed the low mean glycemia group (LMG), whereas the other 9 (mean glycemia above $154 \mathrm{mg} / \mathrm{dL}$ ) were considered the high mean glycemia group (HMG). This division was made considering the American Diabetes Association AlC goal $(<7.0 \%, 53$ $\mathrm{mmol} / \mathrm{mol}$ ) to most adults with diabetes (16).

In addition, the two other variables considered to assess glycemic control were: AlC and glycemic variability $(\mathrm{GV})$, calculated here as the standard deviation (SD) of the 10 consecutive days of measured glycemias.

Polysomnography tests were scored blindly and analyzed by an experienced physician, according to the American Academy of Sleep Medicine manual (20).

Data from the actimeters were processed with $\mathrm{El}$ Temps software (Díez-Noguera, 1, 209+, Barcelona), in order to identify period, amplitude, potency, and acrophase of each subjects' rest/activity rhythm. 
All collected data were analyzed using the following tests: Shapiro-Wilk's W test for normality (data from all variables presented $p$ value greater than 0.05 ); Pearson correlation coefficient, for comparison between variables; T-test for independent samples, when comparing two groups; ANOVA one-way, when comparing data from more than two groups. All results with $p$ value under 0.05 were considered significant. All algorithms used are part of the Statistic software (StatSoft, Inc., 2004, version 6).

\section{Ethics}

The study was approved by the Ethics Committee for Research in Human Beings of Instituto de Ciências Biomédicas, University of São Paulo, number 873/ CEP. It was also approved by all other partner institutions, Associação de Diabetes Juvenil, Hospital das Clínicas e Instituto do Coração da Faculdade de Medicina da Universidade de São Paulo. Informed consent has been obtained from all participants.

\section{RESULTS}

TIDM subjects' age was between 20 and 38 years $(26.3 \mathrm{y} \pm 5.1 \mathrm{y})$. They were non-obese (BMI: $23 \pm 2.9$ $\left.\mathrm{kg} / \mathrm{m}^{2}\right), 8$ were male, and 10 female, and their disease duration was $12.9 \mathrm{y} \pm 7.2 \mathrm{y}$. The nine control subjects' age was between 23 and 38 years $(28.8 \mathrm{y} \pm 5.3 \mathrm{y})$, BMI: $22 \pm 2.7 \mathrm{~kg} / \mathrm{m}^{2}$, and 4 of them were male. Other information concerning TIDM individuals' glycemic control, and sleep parameters from both groups, TIDM and control, are found on table 1 .

\section{Sleep diary}

No difference between the sleep duration of TIDM and control individuals was observed $(p=0.3146)$. Sleep duration was neither correlated with mean glycemia, nor with glycemic variability $(r=0.3043, p=$ $0.2196 ; r=0.1075, p=0.6710$; respectively).
Among our volunteers, 78\% (14 from the TIDM group, and 7 from the control group) presented longer sleep duration during the weekends (Friday to Saturday and Saturday to Sunday) compared to weekdays (Monday to Tuesday, Tuesday to Wednesday, Wednesday to Thursday, and Thursday to Friday). When comparing this sleep duration difference between both groups, the 14 TIDM individuals difference was of $2 \mathrm{~h} 01 \mathrm{~min} 33 \mathrm{~s} \pm 1 \mathrm{~h} 07 \mathrm{~min}$ $19 \mathrm{~s}$, while in the 9 control individuals it was of $57 \mathrm{~min} 17 \mathrm{~s}$ $\pm 36 \mathrm{~min} 24 \mathrm{~s}(p=0.0303)$. The high duration of sleep time on weekend nights or during holidays compared to weekday nights is suggestive of behaviorally induced insufficient sleep, according to Hublin and Sallinen (21).

Sleep quality did not correlate with the mean glycemia, nor with the glycemic variability of TIDM individuals ( $p=0.4381, p=0.4046$, respectively). When the TIDM individuals were separated by the mean glycemia values, only the Low Mean Glycemia group presented significant negative correlation of mean glycemia with sleep quality $(r=-0.8508, p=0.0036)$. No difference between the sleep latency of TIDM and control individuals was observed $(p=0.6347)$. The average of the sleep latency, reported by the individuals during the 10 days, was positively correlated with the glycemic variability $(r=0.6525, p=0.0033$, Figure 1$)$.

\section{Actimeter}

The rest/activity rhythm was analyzed in terms of phases duration, period, amplitude, potency, acrophase, and difference between the observed period and 24 hours. No significant difference was found between the TIDM and control groups in any of these variables.

Night rest duration did not present a significant correlation with mean glycemia $(r=0.5404, p=0.0697)$, whereas it presents a tendency of positive correlation with glycemic variability $(r=0.5706, p=0.0527)$. Interestingly, when considering just the Low Mean Glycemia group, the correlation of the mean glycemia with the night rest duration was negative $(r=-0.8987, p=0.0381)$.

Table 1. Main glycemic control and sleep parameters

\begin{tabular}{|c|c|c|c|c|c|c|c|c|c|}
\hline Groups & HbA1c & $\begin{array}{c}\text { Mean } \\
\text { glycemia } \\
(\mathrm{mg} / \mathrm{dL})\end{array}$ & $\begin{array}{c}\text { Glycemic } \\
\text { variability } \\
\text { (mg/dL) }\end{array}$ & $\begin{array}{c}\text { Mean sleep } \\
\text { duration } \\
(\text { min. })^{\star}\end{array}$ & $\begin{array}{c}\text { Mean sleep } \\
\text { quality* }\end{array}$ & $\begin{array}{l}\text { Mean sleep } \\
\text { latency } \\
\text { (min.)* }\end{array}$ & $\begin{array}{l}\text { PSG sleep } \\
\text { efficiency }\end{array}$ & $\begin{array}{l}\text { PSG arousal } \\
\text { index }\end{array}$ & $\begin{array}{c}\text { PSG apnea/ } \\
\text { Hypopnea } \\
\text { index }\end{array}$ \\
\hline T1DM & $7.8 \pm 1.7$ & $167 \pm 40$ & $74 \pm 17$ & $431 \pm 54$ & $7.1 \pm 1.1$ & $13 \pm 10$ & $90.1 \% \pm 6.7 \%$ & $15.6 \pm 5.9$ & $3.4 \pm 2.1$ \\
\hline Control & - & - & - & $456 \pm 43$ & $7.5 \pm 1.6$ & $15 \pm 9$ & $87.2 \% \pm 6.8 \%$ & $21.6 \pm 14.4$ & $2.9 \pm 2.6$ \\
\hline T-test & - & - & - & ns & ns & ns & ns & ns & ns \\
\hline
\end{tabular}

Mean \pm standard deviation

ns: non-significant difference between both groups.

* Data from the sleep diaries. 


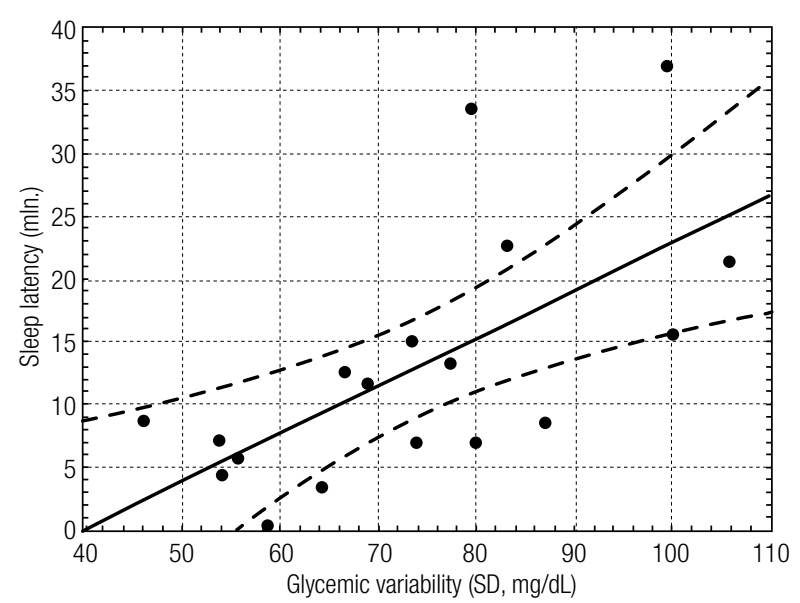

Figure 1. Correlation between glycemic variability and sleep latency $(r=0.6525, p=0.0033)$.

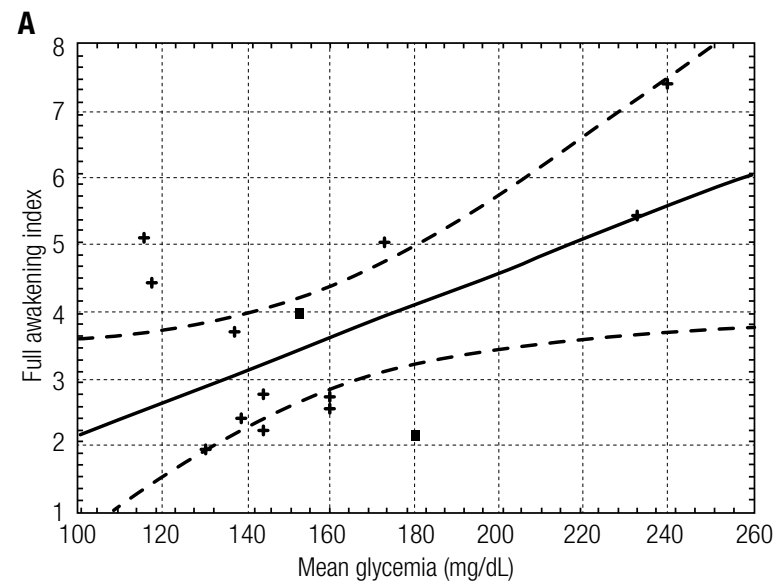

C

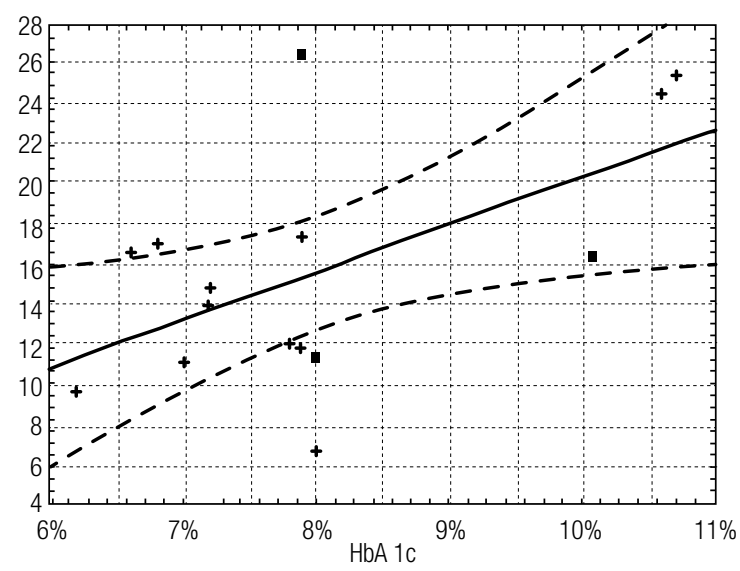

\section{Polysomnography}

None of our volunteers (TIDM and control) presented sleep disorders. The only sleep parameters correlated with the mean glycemia were the full awakenings in$\operatorname{dex}(r=0.5684, p=0.0271$, Figure $2 \mathrm{~A})$ and the apnea-hypopnea index. Surprisingly the correlation with apnea-hypopnea index was negative $(r=-0.5573, p=$ $0.0309)$, as well as a tendency of correlation between apnea-hypopnea index and glycemic variability $(r=$ $-0.4928, p=0.0620$ ). It is possible to observe, though, that this result reflects especially the highest apneahypopnea index of the 5 individuals with lowest mean glycemia $(p=0.0011)$. Similarly to the mean glycemia, AlC was positively correlated with awakenings index $(r$ $=0.6544, p=0.0081$, Figure 2B $)$ and arousal index $(r=$ $0.5680, p=0.0272$, Figure $2 \mathrm{C})$. The $25 \%$ highest glycemic variability TIDM individuals had a significantly higher awakenings index $(p=0.0092$, Figure 2D).

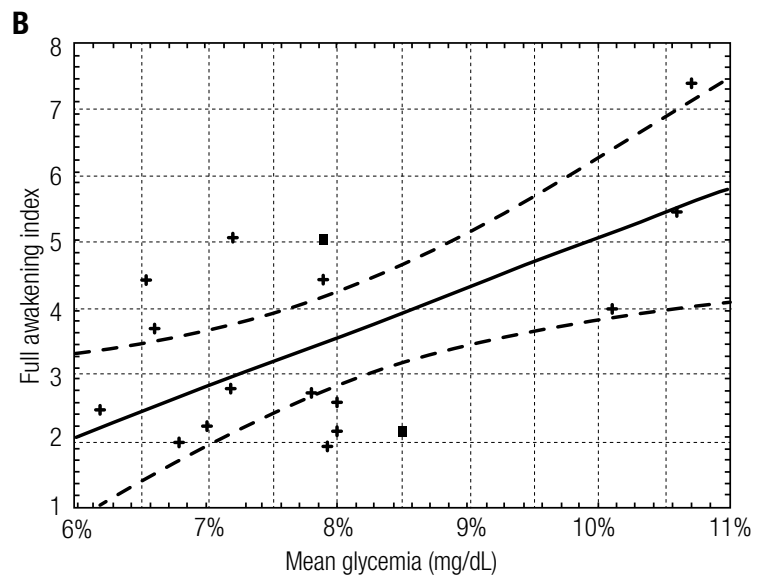

D

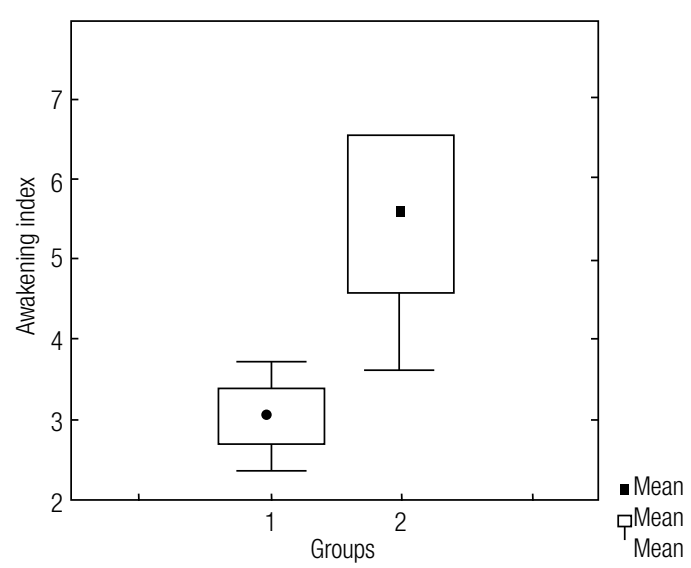

Figure 2. (A-B) Correlation of full awakening index with mean glycemia ( $r=0.5684, p=0.0271)$, and with $\mathrm{A} 1 \mathrm{C}(r=0.6544, p=0.0081)$, respectively; (C) correlation between arousal index and $\mathrm{A} 1 \mathrm{C}(r=0.5680, p=0.0272)$; (D) full awakening index of the $25 \%$ highest glycemic variability (group 2$)$, comparing to the others $(75 \%$, groups 1) $(p=0.0092)$. 
Only one individual presented low glucose levels $(<70 \mathrm{mg} / \mathrm{dL})$ during polysomnography. His glucose stayed low for more than 3 hours. During this time, which was $42.4 \%$ of the total sleep duration, this individual did not wake up, and presented less hypopneas (33.3\%) and awakenings (31.6\%) than during the rest of the night. Additionally, REM sleep was prevalent during this period as seen on figure 3 .

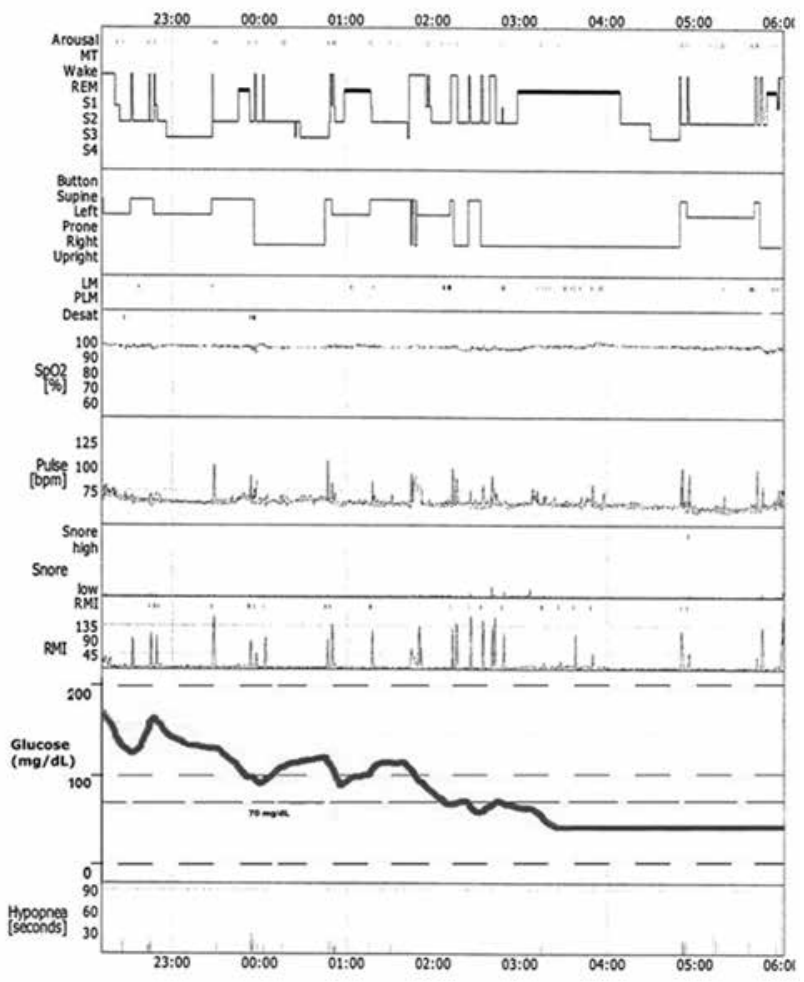

Figure 3. Polysomnography register of the T1DM individual who presented hypoglycemia $(<70 \mathrm{mg} / \mathrm{dL})$ for more than 3 hours during this night.

\section{Epworth Sleepiness Scale}

There was no difference between the control and the TIDM group, nor between High Mean Glycemia and Low Mean Glycemia groups $(p=0.1065, p=0.4791$, respectively). On the other hand the percentage of individuals with a score higher than 10 in the TIDM group was much higher than in the control group $(39 \%$, and $11 \%$, respectively).

\section{DISCUSSION}

The correlations observed between sleep aspects and glycemic control are suggestive of the hypothesized as- sociations. Although our sample size was limited, it was similar to those of previously published studies with TIDM. However, in contrast, we have used a wide range of validated measurement instruments to access sleep parameters, besides more extensive data collection through sleep diaries and actimeters.

In the case of mean sleep latency, a positive correlation with glycemic variability was observed. In other terms, this may be understood as higher difficulty in falling asleep in case of higher glycemic variability. Although this was shown for the first time in our study, it coincides with informal observations both from clinicians and especially from individuals with diabetes, who sometimes report trouble to sleep when experiencing glycemic variations.

The absence of correlation between sleep duration and glycemic control was not surprising, since other groups have reported similar results before $(10,14)$. In our study, differently from findings of other groups that studied TIDM children and adolescents $(10,12)$, and in accordance with a study with TIDM adults (14), sleep duration was not different between TIDM and control subjects. While Pillar and cols. reported in 2003 decreased sleep duration in children with TIDM (12), Yeshayahu and Mahmud reported in 2010 longer sleep durations in adolescents with TIDM (10). The methods used in those two studies were different, whereas the first gauged sleep duration during the polysomnography night, the second considered reported sleep duration. Moreover, there is an age difference that should be considered.

Even though sleep and rest duration were not different between TIDM and control, the duration of sleep extension during weekends was more than twice as long in TIDM patients as it was for control subjects. Taking into account these observations, we speculate that in an environment free of social demands, most of the TIDM individuals would present longer sleep duration than controls, supporting Yeshayahu and Mahmud findings (10).

Moreover, knowing that the sleep extension presented is suggestive of behaviorally induced insufficient sleep (21), which may result of daily sleep deprivation, we understand that the glycemic control in those individuals may be affected. Several authors have reported the negative impact of sleep deprivation, as well as of behaviorally induced insufficient sleep, on insulin resistance and glucose tolerance, as well as learning, memory, attention, immune response, cardiovascular 
function, and neuro-hormonal regulation (21-26). Concerning specifically TIDM, a study revealed that a single partially deprived night is enough to produce insulin resistance (27). In this study individuals were allowed to sleep for 4 hours at night, and in the following day their insulin sensitivity was reduced in 14-21\%.

We consider sleep quality an important parameter, since it was shown, in T2DM, to be negatively correlated with AlC (15). In addition, worse sleep quality was previously reported in individuals with TIDM $(13,14)$, which we did not observe in the present study. In Jauch-Chara and cols.'s study a questionnaire was applied after a polysomnography night (13), whereas our individuals reported their sleep quality on a visual analogue scale every morning. When taking into account ESS score, our results points towards the same direction of Jauch-Chara and cols.'s. (13). While in the control group only one individual ( $11 \%$ of the total) had an ESS score higher than 10, in the TIDM group, 7 individuals (39\% of this group) presented this score level. This finding may be relevant, since a score of 10 points or higher in the ESS is suggestive of low sleep quality and sleep disorders (28). At the same time, although we found no association of mean glycemia or glycemic variability with reported sleep quality, when groups were divided, at least the glycemic parameters of the individuals with a better metabolic control (Low Mean Glycemia group) seemed to be correlated with sleep quality. These results are difficult to interpret, but we suspect that from a certain mean glycemia, sleep quality may not change much, whereas below that level, sleep quality varies in association with glycemic control.

Concerning polysomnography data, differently from other groups $(11,12)$, we did not find sleep disorders in TIDM group. This result is probably due to our strict selection of participants without chronic complications. Mondini and Guilleminault (29), for example, found sleep-disordered breathing in $41.7 \%$ of TIDM individuals. They also reported that this disorder was associated with the presence of neuropathy. Nevertheless, in our study, arousal index and awakenings index were positively correlated with the AlC. This may be understood as an association between these two sleep aspects and glycemic control evident only in a broader angle, which is provided by the 2-3 months of glycemic mean reflected by the AlC value. Therefore, we understand that arousal and awakenings indexes may result of a longer term poor glycemic control. At the same time, we found that the AlC is strongly associated with glycemic variability $(r=0.6228, p=0.0058)$, which, as discussed earlier, may promote sleep changes. These results reveal a significant correlation between the stability or fragmentation of sleep and glycemic control. Moreover, we show that the rate of full awakenings was correlated with the 10 days mean glycemia. Therefore, we conclude that individuals with poor glycemic control have more fragmented sleep, with more arousals and awakenings. At the same time, sleep fragmentation has been associated with increases in insulin resistance and adrenocortical and sympathetic nervous system activity, which, in turn, may hinder the maintenance of good glycemic control $(30,31)$.

The results of higher apnea-hypopnea index observed in individuals with low mean glycemia was surprising. Our results, in this case are just the opposite of another group's (11), who found, in children with TlDM, that the poorer the glycemic control (AlC values) or the greater the disease duration, the greater the apnea index. For this reason, we decided to divide the group, and found that $25 \%$ of the individuals ( 5 individuals) with the lowest GV values (below $59 \mathrm{mg} / \mathrm{dL}$ ) were the ones with the highest apnea-hypopnea in$\operatorname{dex}(4.3 \pm 2.39$ events per hour, comparing to $2.9 \pm$ 1.64). Surprisingly, excluding from this $25 \%$ lowest GV sample the 2 individuals with the highest apneahypopnea index, the remaining 3 individuals present a mean apnea-hypopnea index very similar to the remaining group $(2.7 \pm 1.72)$. And more than that, with this exclusion, the correlation becomes non-significant $(r=$ $-0.2747, p=0.3637)$. Exactly the same happened with the negative correlation between mean glycemia and apnea-hypopnea index, it became non-significant when the two extreme values (AHI > 6) are excluded from the sample $(r=-0.3975, p=0.1786)$. For this reason, although the first results favored a conclusion of higher apnea-hypopnea index in individuals with better metabolic control, it seems that it is just an artifact resulting from 2 individuals' extreme data. It is also worth mentioning that according to the American Academy of Sleep Medicine (32), none of our individuals would be considered to have sleep-disordered breathing, which is diagnosed in cases of at least 15 events per hour, or more than 5 events per hour with clear symptoms.

The difference between stages duration observed by Jauch-Chara and cols. (13), when comparing TIDM with control individuals, was not observed in our study, not even between the different T1DM groups (control vs. TIDM - $\%$ of REM sleep: $21.5 \% \pm 5.3 \%$ vs. $19.9 \% \pm$ 
$7.7 \%, p=0.6216 ; \%$ stage $3,21.7 \% \pm 8.4 \%$ vs. $20.6 \% \pm$ $8.3 \%: p=0.7668)$.

The only case of hypoglycemia during the polysomnography night presented interesting consequences on the sleep parameters. During the hypoglycemia several parameters seemed to become more stable, with more REM sleep, and less hypopneas and awakenings than the rest of the night. Different authors have also found results indicating that the sleep inhibits counter-regulatory responses, and that the hypoglycemia deepens sleep $(12,33)$. These results reinforce the importance of keeping track of glycemia during the night, avoiding hypoglycemia, since if the individual does not wake up, and the glycemia decreases even more, it would put the individual's life in risk $(34,35)$. In addition, we recommend monitoring glucose continuously when performing polysomnography in DM individuals, since, as observed, the occurrence of hypoglycemia may blunt its results.

The results found in this study support at least partially our hypothesis, and are in accordance with results from other groups pointing towards the existence of a vicious circle involving TIDM and sleep (Figure 4). In this vicious circle, poor glycemic control in TIDM affects sleep parameters, which in turn compromises

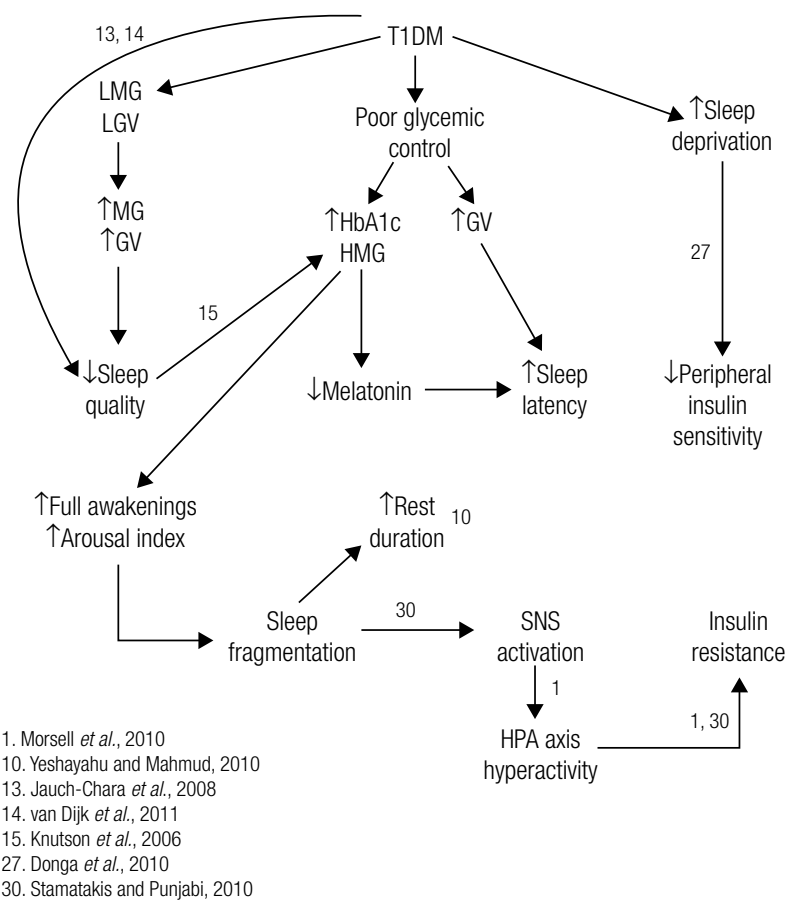

Figure 4. Vicious circle comprising T1DM glycemic control and sleep. the glycemic control. It is similar to the vicious circle that associates sleep with T2DM. Similarly to Knutson and cols.'s proposal for individuals with T2DM (15), we suggest sleep duration and quality to be considered part of T1DM's therapy. Although in the present study we did not isolate variables with the intention to find cause-and-effect relationships, we understand that our results in association to other groups' results make we believe that improving glycemic control may lead to improvements on sleep quality. At the same time, a good night's sleep, with adequate duration and high quality may help to regulate metabolism and sympathoadrenal activity, improving the glycemic control.

We understand that more studies are needed to confirm our findings. Some of the tendencies observed may become significant when analyzing data from more subjects, and unexpected results may be clarified. In addition, more studies are needed to unveil the links connecting glucose metabolism and diabetes with sleep.

Funding: Fundação de Amparo à Pesquisa do Estado de São Paulo (Fapesp) (Grant: 2008/11026-2).

Acknowledgment: we acknowledge support from André Gonçalves, Fernando Fonseca and their team from Faculdade de Medicina do ABC, for their generosity. We thank Lucy Aihara and Cinthya Ugliara for their assistance, and all volunteers for participating in the study and the technicians for their dedicated work.

Disclosure: no potential conflict of interest relevant to this article was reported.

\section{REFERENCES}

1. Leproult R, Balbo M, Spiegel K. Role of sleep duration in the regulation of glucose metabolism and appetite. Best Pract Res Clin Endocrinol Metab. 2010;24:687-702.

2. Tasali E, Leproult R, Ehrmann DA, Van Cauter E. Slow-wave sleep and the risk of type 2 diabetes in humans. Proc Natl Acad Sci U S A. 2008;105:1044-9.

3. Meisinger C, Heier M, Loewel H, Study MKAC. Sleep disturbance as a predictor of type 2 diabetes mellitus in men and women from the general population. Diabetologia. 2005;48:235-41.

4. Mallon L, Broman JE, Hetta J. High incidence of diabetes in men with sleep complaints or short sleep duration: a 12-year follow-up study of a middle-aged population. Diabetes Care. 2005;28:2762-7.

5. Gonzalez-Ortiz M, Martinez-Abundis E, Balcazar-Munoz BR, Pascoe-Gonzalez S. Effect of sleep deprivation on insulin sensitivity and cortisol concentration in healthy subjects. Diabetes Nutr. Metab. 2000;13:80-3.

6. Spiegel K, Tasali E, Leproult R, Van Cauter E. Effects of poor and short sleep on glucose metabolism and obesity risk. Nat Rev Endocrinol. 2009;5:253-61.

7. Hayashino Y, Yamazaki S, Nakayama T, Sokejima S, Fukuhara S. Relationship between diabetes mellitus and excessive sleepiness during driving. Exp Clin Endocrinol Diabetes. 2008;116:1-5. 
8. Pamidi S, Aronsohn RS, Tasali E. Obstructive sleep apnea: role in the risk and severity of diabetes. Best Pract Res Clin Endocrinol Metab. 2010;24:703-15.

9. Barone MTU, Menna-Barreto L. Diabetes and sleep: a complex cause-and-effect relationship. Diabetes Res Clin Pract. 2011;91:129-37.

10. Yeshayahu Y, Mahmud FH. Altered sleep patterns in adolescents with type 1 diabetes: implications for insulin regimen. Diabetes Care. 2010;33:e14.

11. Villa MP, Multari G, Montesano M, Pagani J, Cervoni M, Midulla $F$, et al. Sleep apnoea in children with diabetes mellitus: effect of glycaemic control. Diabetologia. 2000;43:696-702.

12. Pillar G, Schuscheim G, Weiss R, Malhotra A, McCowen KC, Shlitner $A$, et al. Interactions between hypoglycemia and sleep architecture in children with type 1 diabetes mellitus. J Pediatr. 2003;142:163-8.

13. Jauch-Chara K, Schmid SM, Hallschmid M, Born J, Schultes B. Altered neuroendocrine sleep architecture in patients with type 1 diabetes. Diabetes Care. 2008;31:1183-8.

14. van Dijk M, Dinga E, van Dijk JG, Lammers GJ, van Kralingen $K$, Dekkers OM, et al. Disturbed subjective sleep characteristics in adult patients with long-standing type 1 diabetes mellitus. Diabetologia. 2011;54:1967-76.

15. Knutson KL, Ryden AM, Mander BA, Van Cauter E. Role of sleep duration and quality in the risk and severity of type 2 diabetes mellitus. Arch Intern Med. 2006;166:1768-74.

16. American Diabetes Association. Standards of medical care in diabetes--2011. Diabetes Care. 2011;34:S11-61.

17. MonkTH, Reynolds CF, Kupfer DJ, Buysse DJ, Coble PA, Hayes AJ, et al. The Pittsburgh sleep diary. J Sleep Res. 1994;3:111-20.

18. Aitken RCB. Measurement of feelings using visual analogue scales. Proc R Soc Med. 1969;62:989-93.

19. Nathan DM, Kuenen J, Borg R, Zheng H, Schoenfeld D, Heine RJ; for the A1c-Derived Average Glucose (ADAG) Study Group. Translating the $\mathrm{A} 1 \mathrm{C}$ assay into estimated average glucose values. Diabetes Care. 2008;31:1-6.

20. American Academy of Sleep Medicine. The AASM manual for the scoring of sleep and associated events: rules, terminology and technical specifications. American Academy of Sleep Medicine, Westchester, 2007.
21. Hublin C, Sallinen M. Behaviorally induced insufficient sleep. Sleep Med Clin. 2012;7:313-23.

22. Walker MP. Cognitive consequences of sleep and sleep loss. Sleep Med. 2008;9:S29-34.

23. Van Cauter E, Spiegel K, Tasali E, Leproult R. Metabolic consequences of sleep and sleep loss. Sleep Med. 2008;9:S23-8.

24. Spiegel K, Leproult R, Van Cauter E. Impact of sleep debt on metabolic and endocrine function. Lancet. 1999;354:1435-9.

25. Spiegel K, Knutson K, Leproult R, Tasali E, Van Cauter E. Sleep loss: a novel risk factor for insulin resistance and Type 2 diabetes. J Appl Physiol. 2005;99:2008-19.

26. Schmid SM, Hallschmid M, Jauch-Chara K, Wilms B, Lehnert H, Born J, et al. Disturbed glucoregulatory response to food intake after moderate sleep restriction. Sleep. 2011;34:371-7.

27. Donga $E$, van Dijk M, van Dijk JG, Biermasz NR, Lammers GJ, van Kralingen K, et al. Partial sleep restriction decreases insulin sensitivity in type 1 diabetes. Diabetes Care. 2010;33:1573-7.

28. Johns MW. A new method for measuring daytime sleepiness: the Epworth sleepiness scale. Sleep. 1991;14:540-5.

29. Mondini S, Guilleminault C. Abnormal breathing patterns during sleep in diabetes. Ann Neurol. 1985;17:391-5.

30. Stamatakis KA, Punjabi NM. Effects of sleep fragmentation on glucose metabolism in normal subjects. Chest. 2010;137:95-101.

31. Clarenbach CF, West SD, Kohler M. Is obstructive sleep apnea a risk factor for diabetes? Discov Med. 2011;12:17-24.

32. American Academy of Sleep Medicine. The international classification of sleep disorders, 2nd ed. American Academy of Sleep Medicine, Westchester, 2005.

33. Banarer S, Cryer PE. Sleep-related hypoglycemia-associated autonomic failure in type 1 diabetes: reduced awakening from sleep during hypoglycemia. Diabetes. 2003;52:1195-203.

34. Schultes B, Jauch-Chara K, Gais S, Hallschimd M, Reiprich E, Kern W, et al. Defective awakening response to nocturnal hypoglycemia in patients with type 1 diabetes mellitus. PLoS Med. 2007;4:e69.

35. Gill GV, Woodward A, Casson IF, Weston PJ. Cardiac arrhythmia and nocturnal hypoglycaemia in type 1 diabetes--the 'dead in bed' syndrome revisited. Diabetologia. 2009;52:42-5. 


\section{Sleep and glycemic control in type 1 diabetes}

\section{Mark Thomaz Ugliara Barone, Daniela Wey, Fabiola Schorr, Denise Reis Franco, Mario Kehdi Carra, Geraldo Lorenzi-Filho, Luiz Menna-Barreto}

Arch Endocrinol Metab. 2015;59(1):71-8

DOI: 10.1590/2359-3997000000052

Where you read:

C

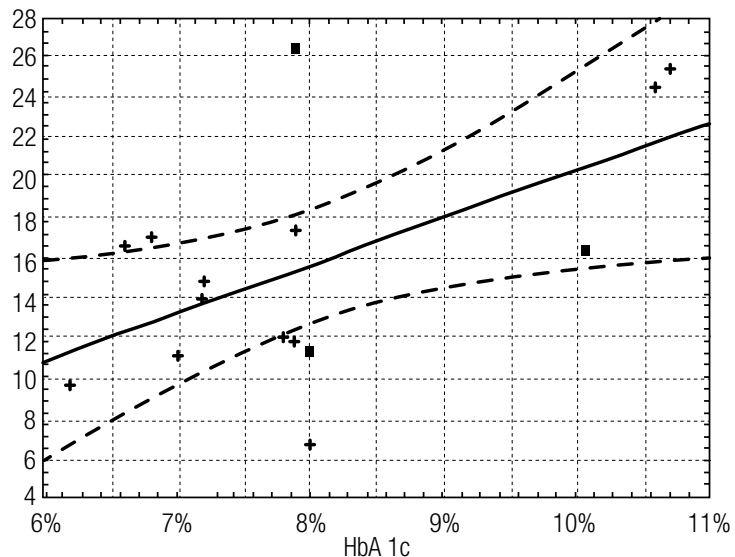

Figure 2. (A-B) Correlation of full awakening index with mean glycemia $(r$ $=0.5684, p=0.0271)$, and with A1C $(r=0.6544, p=0.0081)$, respectively; (C) correlation between arousal index and A1C ( $r=0.5680$, $p=0.0272) ;(\mathbf{D})$ full awakening index of the $25 \%$ highest glycemic variability (group 2), comparing to the others $(75 \%$, groups 1$)(p=$ 0.0092).

Where you read:

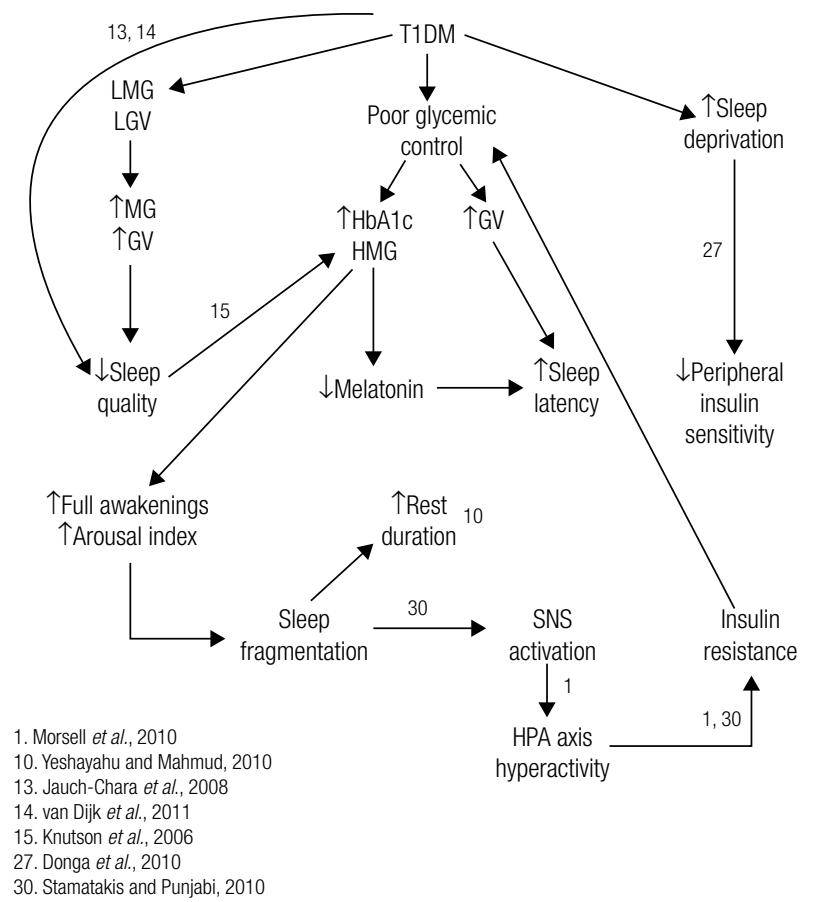

Figure 4. Vicious circle comprising T1DM glycemic control and sleep.
Should read:

C

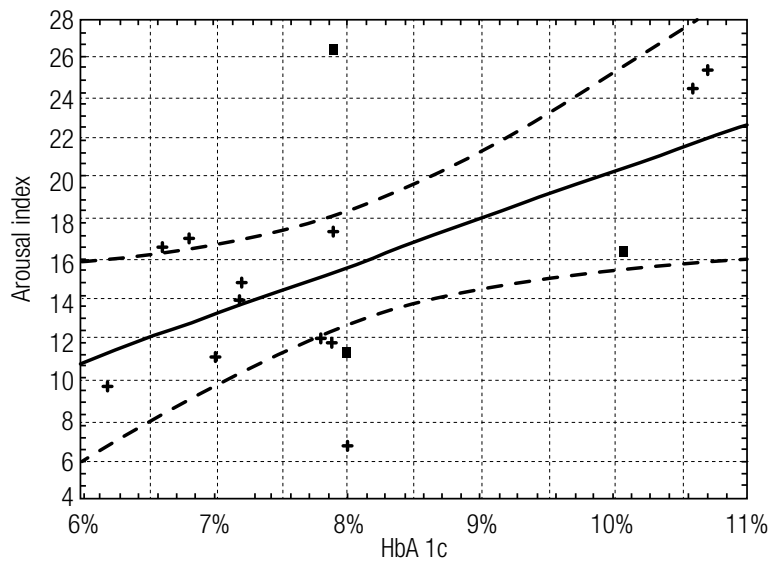

Figure 2. (A-B) Correlation of full awakening index with mean glycemia ( $r$ $=0.5684, p=0.0271)$, and with A1C $(r=0.6544, p=0.0081)$, respectively; (C) correlation between arousal index and A1C ( $r=0.5680$, $p=0.0272$ ); (D) full awakening index of the $25 \%$ highest glycemic variability (group 2$)$, comparing to the others $(75 \%$, groups 1$)(p=$ 0.0092).

Should read:

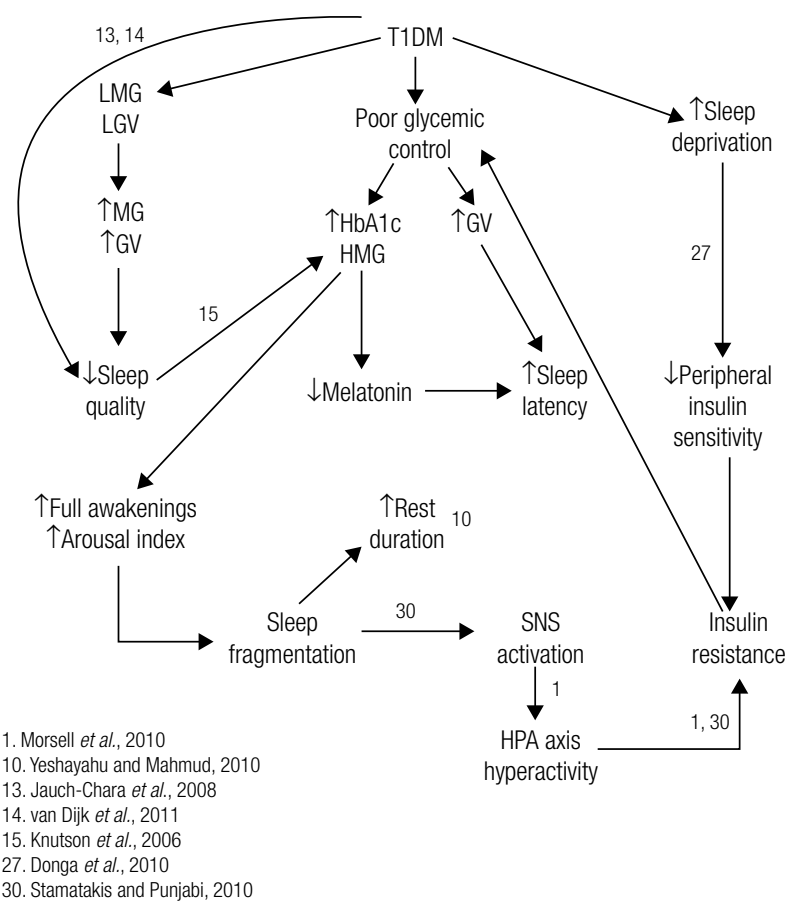

Figure 4. Vicious circle comprising T1DM glycemic control and sleep. 